\title{
Implication of a Novel Function of Sar1 in the Nucleus
}

\author{
Kotaro Horikawa ${ }^{1}$, Tomohiro Yorimitsu ${ }^{1}$, Chie Kodera ${ }^{2}$, and Ken Sato ${ }^{1,2^{*}}$ \\ ${ }^{1}$ Department of Life Sciences, Graduate School of Arts and Sciences, University of Tokyo, Komaba, Meguro-ku, \\ Tokyo 153-8902, Japan, ${ }^{2}$ Department of Biological Sciences, Graduate School of Science, University of Tokyo, \\ Hongo, Bunkyo-ku, Tokyo 113-0033, Japan
}

\begin{abstract}
The coat protein complex II (COPII) generates transport carriers that deliver newly synthesized proteins from the endoplasmic reticulum (ER) to the Golgi apparatus. The small GTPase Sar1 is a well-known regulator of the assembly of the COPII coat. In the present study, we demonstrate that, besides its well-established role in ER-to-Golgi trafficking, the nuclear localization of Sar1 is essential for the viability of Saccharomyces cerevisiae. Inhibition of either the nuclear entry or retention of Sar1 leads to a severe growth defect. Additionally, in vivo deletion of Sar1, by using conditional genetic depletion, further demonstrates that the loss of nuclear localization of Sar1 results in an abnormal nuclear envelope shape. Our findings highlighted a possible novel role of Sar1 within the nucleus, which may relate to the proper formation of the nuclear envelope.
\end{abstract}

Key words: Sar1, COPII, small GTPase, nuclear envelope, membrane traffic

\section{Introduction}

Vesicular traffic from the endoplasmic reticulum (ER) to the Golgi apparatus is thought to occur at specialized regions of the ER, known as ER exit sites (ERES). In these distinct regions of the ER, the components of the coat protein complex II (COPII) generate COPII-coated transport carriers (COPII vesicles) through a sequence of events (Barlowe and Miller, 2013; Gomez-Navarro and Miller, 2016). Formation of vesicles at ERES is initiated by the activation of the small GTPase Sar1 through the conversion of its GDP to GTP (Nakano and Muramatsu, 1989), and it is mediated by the ER-resident Sec12 guanine nucleotide exchange factor (GEF) (Barlowe and Schekman, 1993). This GDP-GTP transition triggers the exposure of the Nterminal amphipathic helix element of Sar1 that inserts into the ER membrane (Huang et al., 2001; Bi et al., 2002). Following the binding of the Sar1-GTP to the inner coat Sec23/24 complex via the Sec23 subunit, the Sec24 subunit captures the cargo to form a prebudding complex (Miller et al., 2002; Mossessova et al., 2003; Miller et al., 2003). The Sec23 subunit is the GTPase-activating protein (GAP) for Sar1 (Yoshihisa et al., 1993), and repeated cycles of Sec12dependent GTP loading to Sar1 and Sec23-mediated

\footnotetext{
*To whom correspondence should be addressed: Ken Sato, Department of Life Sciences, Graduate School of Arts and Sciences, University of Tokyo, Komaba, Meguro-ku, Tokyo 153-8902, Japan.

Tel: +81-3-5454-6749

E-mail: kensato@bio.c.u-tokyo.ac.jp
}

hydrolysis facilitate proper and efficient cargo sorting into COPII vesicles (Sato and Nakano, 2005). Subsequently, the pre-budding complex recruits the outer shell of the Sec13/31 complex, which in turn polymerizes adjacent prebudding complexes to drive membrane deformation and vesicle budding (Bi et al., 2007; Tabata et al., 2009). Scission of vesicle is also aided by the N-terminal amphipathic helix of Sar1 (Lee et al., 2005). In this manner, Sar1 plays a key role in directly generating COPII-coated transport carriers on the ER membrane.

We previously showed that the Sec23 homolog Nel1 in Saccharomyces cerevisiae exhibits strong Sar1-GAP activity but lacks the ability to function as a subunit of COPII. Our data also indicated that a subpopulation of Nel1 shuttles to the nucleus (Kodera et al., 2014). On the basis of these findings, we hypothesized that a fraction of Sar1 would also be basally localized inside the nucleus and may play some functional roles.

Here, we report that the nuclear localization of Sar1 is essential for yeast cell growth, in addition to its indispensable role in protein trafficking from the ER to the Golgi apparatus. Inhibition of either the nuclear entry or nuclear retention of Sar1 caused a severe growth defect. We also demonstrate, by conditional depletion of Sar1, that the lack of nuclear localization of Sar1 leads to an abnormal nuclear envelope shape. Our findings propose a possible novel role of Sar1 within the nucleus, which may be independent of its vesicular trafficking function. 


\section{Materials and Methods}

\section{Yeast strains and media}

S. cerevisiae strains used in this study were ANY21 (MATa ura3-52 leu2-3,112 trp1-289 his3 his4 suc gal2) (Nakano et al., 1988), KHY1 (MATa sar1::HIS3 pep4::ADE2 ura3 leu2 trp1 his3 ade2 lys2 /pSAR1/316(pRS316[SAR1 URA3])), yTHC5854 (BY4741 (MATa his3- $\Delta 1$ leu2- $\Delta 0$ ura3- $\Delta 0$ met15- $\Delta 0)$ URA3::CMV-tTA prmSAR1::KAN-tetO -TATA $_{C Y C 1}$ ) (Open Biosystems, Huntsville, AL, USA), YTY189 (yTHC5854 his3$\Delta 1: \because p R S 303)$, YTY190 (yTHC5854 his3- $1: \because p R S 303-S A R 1)$, KHY2 (yTHC5854 his3- $1:: p R S 303-S A R 1-N E S$ ), and KHY3 (yTHC5854 his3- $1: \because p R S 303-S A R 1-N E S m t)$. KHY1 was obtained from TOY221 (Saito et al., 1999) by plasmid curing. Yeast strains were grown at $30^{\circ} \mathrm{C}$ in YPD medium $(1 \%$ Bacto-yeast extract (nacalai tesque, Inc., Kyoto, Japan), $2 \%$ polypeptone (Nihon Seiyaku), and $2 \%$ dextrose) or MCD medium $(0.67 \%$ yeast nitrogen base without amino acids (FORMEDIUM, Norfolk, UK), $0.5 \%$ casamino acids, and $2 \%$ dextrose) with the appropriate supplements. If necessary, 5-fluoroorotic acid (5-FOA) (Wako, Tokyo, Japan) and doxycycline (Dox) (Merck Millipore, Billerica, MA, USA) were used at final concentration of $0.1 \%$ and 10 $\mu \mathrm{g} / \mathrm{ml}$, respectively.

\section{Antibodies}

The following antibodies were used for immunoblotting: antiCPY (A-6428, Thermo Fisher, Bellefonte, PA, USA), anti-Pgk1 (459250, Thermo Fisher, Bellefonte, PA, USA), anti-Sar1 (Kodera et al., 2011), anti-GFP (632381, Clontech, Mountain View, CA, USA), anti-Dpm1 (A6429, Invitrogen, Carlsbad, CA, USA), antiPom152 (MAB5388, Abnova, Walnut, CA, USA).

\section{Plasmids}

The plasmids expressing Htb1-GFP (pHTB1-GFP/314), Sar1 (pSAR1/316, pSAR1-BglII-2(316) or pSAR1/314), Sar1-mCherry (pSAR1-mCherry/316 or pSAR1-mCherry/314), and Sec71mCherry (pSec71-mCherry/315) have been described previously (Yorimitsu and Sato, 2012; Kakoi et al., 2013; Kodera et al., 2014). To generate the Sar1-mCherry-2NLS and Sar1-NES expression plasmids, fragments encoding Sar1-mCherry-2NLS and Sar1-NES, together with upstream flanking regions, were amplified by PCR from pSAR1-mCherry/316 and pSAR1/316, respectively. The gene of SAR1 in pSAR1-BglII-2(316) was replaced with SAR1-mCherry-2NLS fragment using the BamHI and $B g l$ II sites, yielding pSAR1-mCherry-2NLS/316. SAR1-NES fragment was inserted into $S a c I$ and $X h o I$ sites of pRS314, yielding pSAR1-NES/314. The plasmids expressing $\Delta 23-$ Sar1mCherry-2NLS (p $\Delta 23-S A R 1-m C h e r r y-2 N L S / 316)$ and Sar1NESmt (pSAR1-NESmt/314) were generated by primer-directed mutagenesis using pSAR1-mCherry-2NLS/316 and pSAR1$\mathrm{NESmt} / 314$ as the template, respectively.

\section{Subcellular fractionation}

Subcellular fractionation was performed as described previously (Vida et al., 1990). In brief, wild-type cells (ANY21) were grown to mid-log phase and were converted to spheroplasts by Zymolyase treatment. Spheroplasts were resuspended in $2 \times$ lysis buffer (40 mM HEPES-KOH, pH 7.4, 400 mM sorbitol, 100 mM KOAc, 4 mM EDTA, supplemented with protease inhibitor cocktail (PIC; Roche) and $1 \mathrm{mM}$ DTT), and then centrifuged at 3,000 rpm for 10 min in a Beckman JA-14 rotor. The spheroplast pellets were resuspended in lysis buffer (20 mM HEPES-KOH, pH 7.4, 200 $\mathrm{mM}$ sorbitol, $50 \mathrm{mM}$ KOAc, $2 \mathrm{mM}$ EDTA, supplemented with PIC and $1 \mathrm{mM}$ DTT), and then subjected to 7 strokes in a Dounce homogenizer. The lysates were centrifuged at $300 \times \mathrm{g}$ for $5 \mathrm{~min}$ to remove unlysed cells, yielding total fractions. The total fractions were centrifuged at $13,000 \times \mathrm{g}$ for $15 \mathrm{~min}$ to generate S13 supernatants and P13 pellets. P13 pellets were washed four times with lysis buffer. P13 was enriched for nucleus and membrane organelles. For further analysis of protein localization, $\mathrm{P} 13$ pellets were resuspended in B88 (20 mM HEPES-KOH, pH 6.8, $250 \mathrm{mM}$ sorbitol, $150 \mathrm{mM} \mathrm{KOAc,} 5 \mathrm{mM} \mathrm{MgOAc}$ ). The samples were incubated with $1 \mathrm{M} \mathrm{NaCl}, 0.1 \% \mathrm{NP}-40$, or $0.1 \%$ Triton X-100 in B88, respectively, and washed with the same buffer. After each treatment, samples were centrifuged at $15,000 \mathrm{rpm}$ for $5 \mathrm{~min}$ in a HITACHI T15A61 rotor to separate soluble and insoluble fractions. The samples were analyzed by SDS-PAGE and immunoblotting.

\section{Western blotting and quantification}

Samples were separated by SDS-PAGE and transferred to 0.45$\mu \mathrm{m}$ PVDF membranes (Millipore, Billerica, MA, USA). Western blots were developed using the ECL Plus (GE Healthcare Japan Ltd., Tokyo, Japan). Bands were imaged and quantified by LAS-3000 (Fujifilm, Tokyo, Japan).

\section{Fluorescence microscopy}

Yeast cells expressing fluorescent protein-fused proteins were grown to mid-log phase at $30^{\circ} \mathrm{C}$. Fluorescence microscopy observations were carried out using an Olympus IX71 microscope (Olympus, Tokyo, Japan) equipped with a CSU10 spinning-disk confocal scanner (Yokogawa Electric Corporation, Tokyo, Japan) and an electron-multiplying charge-coupled device camera (iXon, DV897; Andor Technology, South Windsor, CT, USA). The acquired images were analyzed by Andor iQ (Andor Technology, South Windsor, CT, USA). In this setting, a $473 \mathrm{~nm}$ solid-state laser (J050BS; Showa Optronics, Tokyo, Japan) was used to excite GFP and a $561 \mathrm{~nm}$ solid-state laser (J050YS; Showa Optronics, Tokyo, Japan) was used to excite mCherry. 


\section{Results}

\section{A fraction of Sar1 appears to be localized on the inner nuclear membrane.}

Molecules with molecular weight greater than 40-60 kDa have difficulty entering the nucleus through the nuclear pore complex by passive diffusion (Okuno et al., 1993). Since the molecular weight of endogenous Sar1 (at 21.5 $\mathrm{kDa}$ ) is lower than the cutoff mass blocking the diffusion of molecules into the nucleus through nuclear pores, a functional nuclear localization signal (NLS) would not be required for its active import into the nucleus. To confirm this, yeast cells were converted into spheroplasts, homogenized, and the extracts were separated by centrifugation into low-speed supernatant (S13) and low-speed pellet (P13) fractions (Fig. 1A) (Vida et al., 1990). Under these conditions, the nuclear marker Htb1-GFP (histone H2B) and the ER membrane marker Dpm1 were detected in the P13 fraction. The soluble marker protein Pgk1 was detected exclusively in the S13 fraction, indicating complete cell lysis. A significant portion of endogenous Sar1 was recovered in the S13 fraction, but it was also fractionated into the $\mathrm{P} 13$ fraction (percent ratio of $\mathrm{P} 13 /$ total $=7.6 \pm 2.5 \%$ ) (Fig. 1B). It is assumed that after the cell is lysed, Sar1 is likely to be separated from Sec12 and Sec23/24, and its endogenous GTPase activity would lead to its dissociation from membrane. In contrast, when a fusion of Sar1 with mCherry at its C-terminus (Sar1-mCherry, (Yorimitsu and Sato, 2012; Kakoi et al., 2013)) was exogenously expressed, it was found to be predominantly localized to the S13 fraction (Fig. 1A), and $~ 12$-fold less Sar1-mCherry was recovered in the $\mathrm{P} 13$ fraction (percent ratio of $\mathrm{P} 13 /$ total $=0.61 \pm 0.54 \%$ ) (Fig. 1B). These results suggest that passive diffusion of the Sar1 protein through the nuclear pore complexes was blocked by the addition of the Cterminal mCherry-tag, because the size of the Sar1mCherry fusion protein is sufficiently large to preclude passive diffusion into the nucleus, leading us to propose that Sarl detected in the P13 fraction partly reflects its nuclear localization. As a control for the detection of nuclear-targeted Sar1, we examined the localization of Sar1-mCherry with two tandem repeats of the NLS from the T-antigen of SV40 at its C-terminus (Sar1mCherry-2NLS) (Fig. 1B) (Kalderon et al., 1984; Kosugi et al., 2009). Although Sar1-mCherry-2NLS was detected both in the S13 and P13 fractions, it was $\sim 95$-fold more abundant in the P13 fraction (percent ratio of P13/ total $=58 \pm 29 \%$ ) than that observed with Sar1-mCherry, supporting our observation that Sar1 found in the P13 fraction partly reflects its nuclear localization.

The association of Sar1 with the P13 fraction was further analyzed by chemical treatment of P13 before centrifugation (Fig. 1C). Incubation of the P13 fraction in $1 \mathrm{M} \mathrm{NaCl}$ or a weak detergent $(0.1 \%$ NP- 40$)$ failed to release Sar1 into the soluble fraction. The nearly complete extraction of Sar 1 from the sedimenting fraction by using $0.1 \%$ Triton $\mathrm{X}-100$, along with the known nuclear pore complex marker Pom152, suggests that this pellet association was not merely a result of protein aggregation. These results indicate that the endogenous Sarl observed in the P13 fraction adheres tightly to the membrane.

We also analyzed the localization of Sar1mCherry-2NLS in living cells (Fig. 1D). Consistent with our fractionation results, confocal images revealed cortical ER and perinuclear staining of Sar1-mCherry-2NLS, whereas no obvious signal was detected in the nucleoplasmic region. Sar1 has been shown to anchor to the membrane via its N-terminal amphipathic helix (Lee et al., 2005), and truncation of this 23-residue helical domain in Sar1-mCherry-2NLS ( $\triangle 23$-Sar1-mCherry-2NLS) resulted in strong nucleoplasmic accumulation (Fig. 1E), confirming that the NLS attached to the Sar1-mCherry is functional. Together, these results imply that Sar1 is able to passively diffuse into the nucleus and localize along the periphery of the inner nuclear membrane, in addition to its known localization in the ER.

\section{Nuclear localization of Sar1 is required for normal cell viability.}

To determine the physiological importance of nuclear localization of Sar1, we tested whether yeast cells that express endogenous levels of Sar1-mCherry or Sar1 fused with the nuclear export signal (NES) sequence of a protein kinase inhibitor (Wen et al., 1995) (Sar1-NES) as the sole copy of $S A R 1$ could grow in the absence of a chromosomally encoded copy of $S A R 1$. A sarl $\triangle$ strain maintained by a wildtype copy of $S A R 1$ on a URA3 plasmid was transformed with an empty vector (TRP1 plasmid) or the same vector encoding wild-type Sar1, Sar1-mCherry, or Sar1-NES. As shown in Fig. 2A, all the cells grew equally well on control plates in the presence of the wild-type Sar1 maintenance plasmid. In contrast, when cells were grown on 5fluoroorotic acid (5-FOA) to eliminate the wild-type Sar1 maintenance plasmid, cells expressing the Sar1-mCherry that is unable to enter the nucleus grew weakly. Furthermore, cells expressing Sar1-NES were unable to grow in the absence of the wild-type Sar1 protein. This effect is not likely due to the steric bulk of the NES moiety, since point mutation of the critical residues within the NES (Wen et al., 1995) (Sar1-NESmt) almost completely abolished the growth inhibitory activity (Fig. 2B). These results clearly show that the nuclear localization of Sarl is required for normal cell growth.

\section{The absence of Sar1 in the nucleus leads to abnormal nuclear envelope morphology.}

To assess the functional role of Sar1 in the nucleus, we 
A

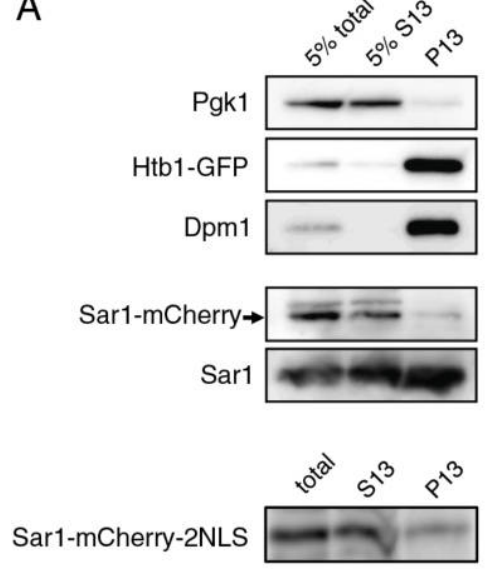

B

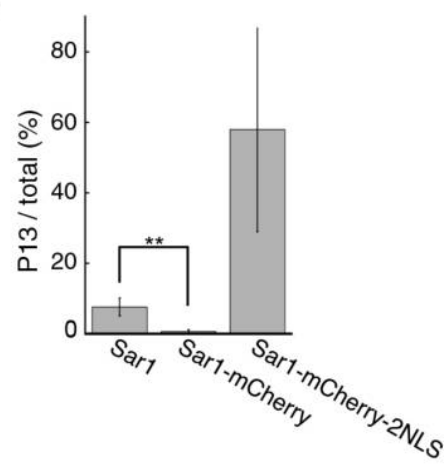

C

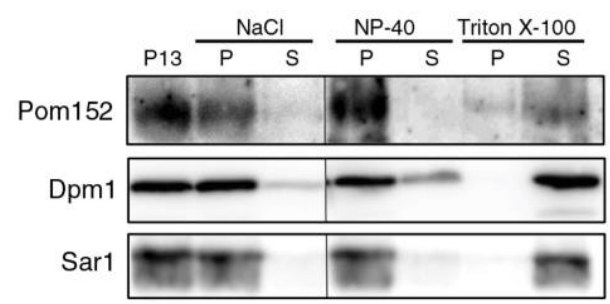

D

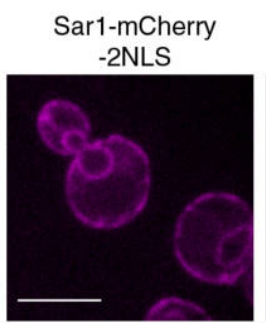

Htb1-GFP
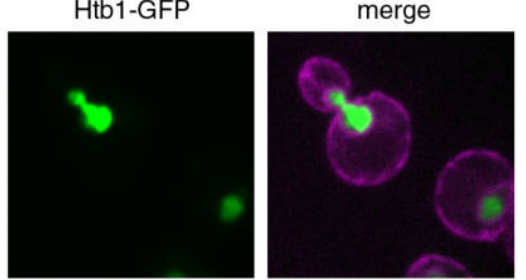

E

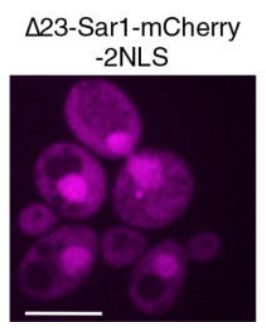

Htb1-GFP
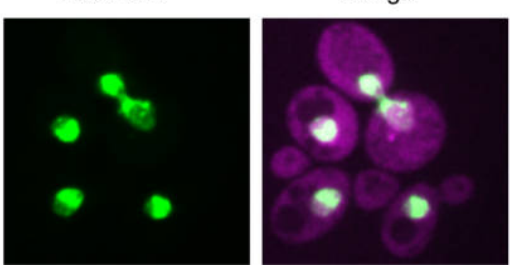

Fig. 1. Subcellular localization of Sar1. (A) Subcellular fractionation analyses of Sar1. Cells (ANY21) expressing Htb1-GFP and Sar1-mCherry (or Sar1mCherry-2NLS) were grown to mid-log phase, converted to spheroplasts, homogenized, and centrifuged at $13,000 \times \mathrm{g}$. Five percent of the total homogenate, $5 \%$ of the supernatant (S13), and the entire pellet (P13) fractions were analyzed by SDS-PAGE and immunoblotting. The total homogenate and total supernatant were used to estimate Sar1-mCherry-2NLS. The grouping of blots cropped from different parts of the same gel or from different gels. (B) Quantification of Sar1, Sar1-mCherry, and Sar1-mCherry-2NLS levels in the P13 fraction. The percentages of Sar1, Sar1-mCherry, and Sar1mCherry-2NLS in the P13 fraction were quantified by immunoblotting as in (A). The means and standard deviations of three independent experiments are shown in the graph. These results were verified by applying the Student's t-test. **P value was $<0.01$. (C) The association of Sar1 with the P13 fraction as assessed by chemical treatment. P13 fractions were treated with $1 \mathrm{M} \mathrm{NaCl}$ for $15 \mathrm{~min}, 0.1 \% \mathrm{NP}-40$, or $0.1 \%$ Triton X-100 for $30 \mathrm{~min}$ on ice and centrifuged at 15,000 rpm. The resulting pellet $(\mathrm{P})$ and supernatant $(\mathrm{S})$ were analyzed by SDS-PAGE and immunoblotting. The grouping of blots cropped from different parts of the same gel or from different gels. Dividing lines between blots represent noncontiguous gel lanes. (D, E) Localization of Sar1mCherry-2NLS and $\Delta 23$-Sar1-mCherry-2NLS. Cells expressing Htb1-GFP and Sar1-mCherry-2NLS (D) or $\Delta 23-$ Sar1-mCherry-2NLS (E) were grown to mid-log phase and observed by confocal microscopy. (D, E) Scale bars, $5 \mu \mathrm{m}$. 


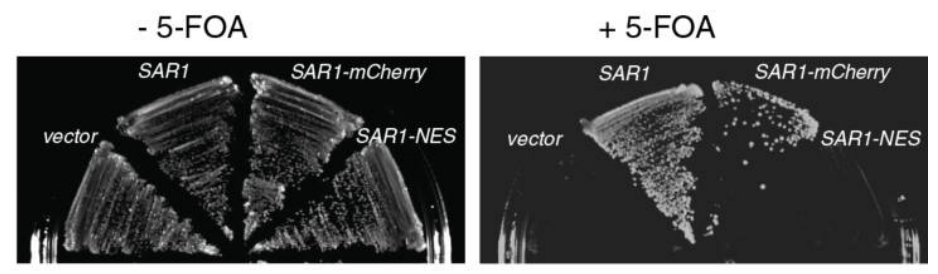

B $\operatorname{sar1} \triangle$ SAR1(URA3)
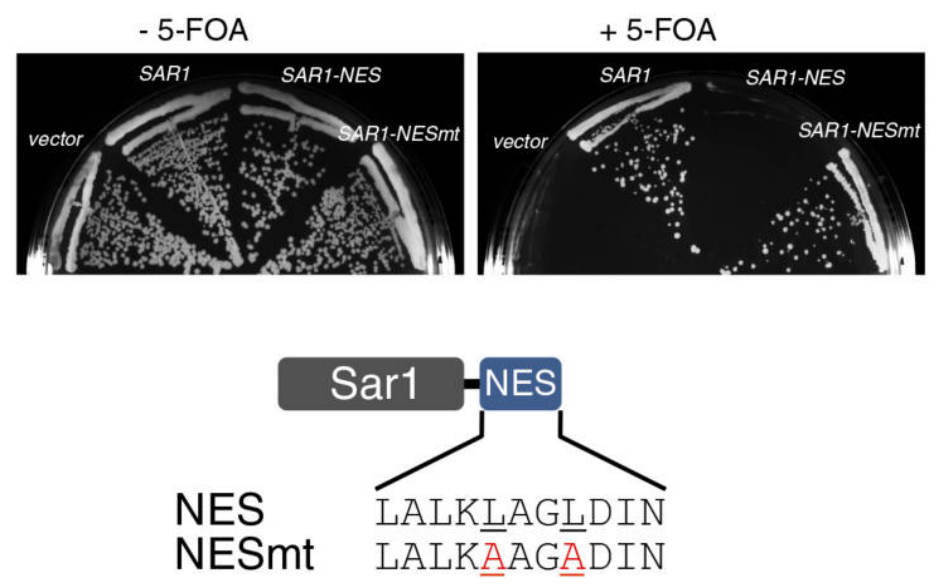

Fig. 2. Nuclear localization of Sar1 is necessary for cell viability. (A) Complementation analysis of a sar $1 \Delta$ strain. sar1 $1 \Delta$ cells (KHY1) expressing Sar1 from a URA3 plasmid were transformed with an empty vector (TRP1), or the same vector encoding wild-type Sar1, Sar1-mCherry, or Sar1-NES. Transformants were streaked onto plates containing 5-FOA and incubated at $30^{\circ} \mathrm{C}$. (B) sarl $1 \Delta$ cells expressing Sar1 from a URA3 plasmid were transformed with an empty vector (TRP1), or the same vector encoding wild-type Sar1, Sar1-NES, or Sar1-NESmt. Transformants were streaked onto plates containing 5-FOA and incubated at $30^{\circ} \mathrm{C}$ (upper panels). Lower panel show the nuclear export signal (NES) sequence of a protein kinase inhibitor fused to Sar1. NES mutant (NESmt) contains alanine substitutions (underlined) replacing conserved hydrophobic residues (shown in red).

used a conditional Sar1 depletion strain (tetO-SAR1), which carries a doxycycline-repressible allele of the SAR1 gene. In the tetO-SAR1 strain, we chromosomally integrated vector or plasmid expressing wild-type Sar1, Sar1-NES, or Sar1-NESmt under the control of the native promoter. The expression of Sarl protein in tetO-SARI cells in the presence of doxycycline (Dox) was markedly reduced as compared to that in the absence of Dox (Fig. 3A, labeled "-"), whereas the expression from the chromosomally integrated Sarl (Fig. 3A, labeled "SARl") and its derivatives (Fig. 3B, labeled "SAR1-NES" or "SAR1-NESmt") was not affected.

By using these tetO-SAR1 cells, the nuclear envelope membranes were visualized by expressing plasmid encoding Sec71-mCherry (Fig. 3C, D). In the absence of Dox, all the cells exhibited normal nuclear envelope morphology (indicated by arrowheads). Upon Dox treatment of tetOSAR1 cells expressing either wild-type Sar1 or Sar1NESmt, no effect was observed on the morphology of the nuclear envelope, with both types of cells were expected to express Sar1 in the cytosol and the nucleus. In contrast, Dox treatment of tetO-SAR1 cells, which lack Sar1 both in the cytosol and the nucleus, caused disruptive effects on the morphology of the nuclear envelope. Notably, Dox-treated tetO-SAR1 cells expressing Sar1-NES, which is expected to express Sar1 in the cytosol but not in the nucleus, also showed abnormal morphology of the nuclear envelope. These results suggest that Sar1 localization inside the nucleus is a necessary pre-requisite for proper nuclear envelope formation. We also attempted to observe the nucleus with Htb1-GFP, but tetO-SAR1 cells failed to express the Htb1-GFP, even in the absence of Dox treatment, for unknown reasons.

To determine if these defects in nuclear envelope morphology correlate with vesicular trafficking, we examined the intracellular trafficking of carboxypeptidase Y (CPY) within the same set of tetO-SAR1 cells as in Fig. 3C. We observed that the Dox-treated tetO-SAR1 cells (Fig. 3E, labeled "-") accumulated the ER-specific p1 precursor form of CPY, but the cells expressing chromosomally integrated wild-type Sarl did not (Fig. 3E, labeled "SARl"), thereby confirming that the Dox-mediated Sar1 depletion significantly blocks the ER-to-Golgi transport. Contrary to 

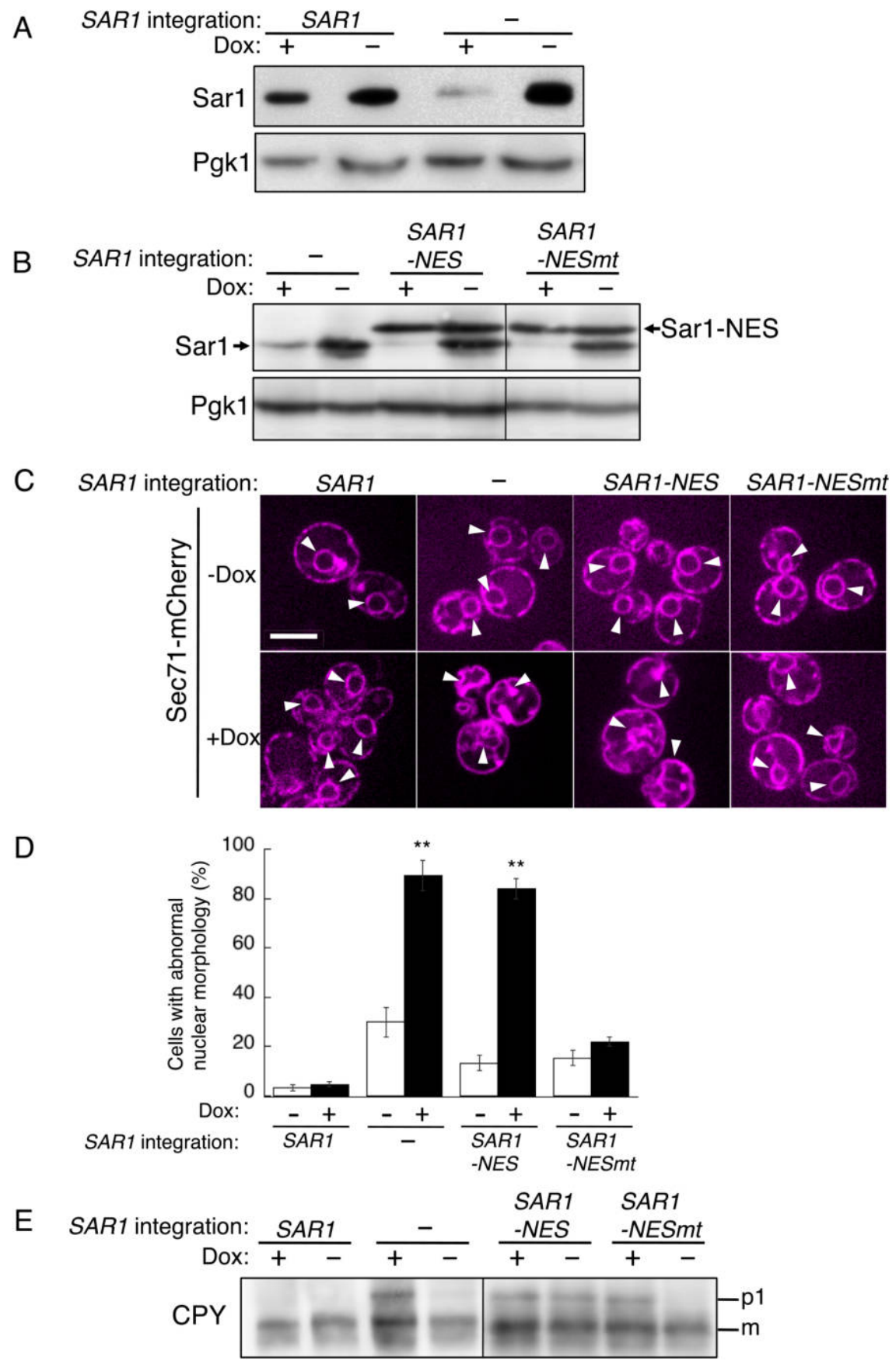

Fig. 3. Sar1 localization in the nucleus is required for the proper formation of the nuclear envelope. (A, B) Whole-cell lysates prepared by glass beads homogenization from tetO-SAR1 cells with the chromosomally integrated vector (-) or plasmid expressing wild-type Sar1 (SAR1), Sar1-NES (SAR1-NES), or Sar1-NESmt (SAR1-NESmt) treated for $4 \mathrm{~h}$ with $(+)$ or without $(-)$ Dox were analyzed by immunoblotting. Pgk1 serves as loading control (lower panels). The grouping of blots was cropped from the same gel for each protein. Dividing lines between blots represent noncontiguous gel lanes. (C) tetOSAR1 cells with the chromosomally integrated vector (-) or plasmid expressing wild-type Sar1 (SAR1), Sar1-NES (SAR1-NES), or Sar1-NESmt (SAR1$N E S m t)$ were transformed with the Sec71-mCherry expression plasmid and treated for $4 \mathrm{~h}$ with $(+)$ or without (-) Dox, and then observed by confocal microscopy. Arrowheads indicate nuclear membrane. Scale bar, $5 \mu \mathrm{m}$. (D) Cells were treated as in (C), and the percentages of cells with abnormal nuclear morphology were determined in three independent experiments ( $>50$ cells were analyzed for each condition). The means and standard deviations are shown in the graph. These results were verified by applying the Student's t-test. ${ }^{*}$ P value was $<0.01$. (E) tetO-SARI cells with the chromosomally integrated vector (-) or plasmid expressing wild-type Sar1 (SAR1), Sar1-NES (SAR1-NES), or Sar1-NESmt (SAR1-NESmt) were grown to log phase and equal amounts of whole cell lysates were subjected to immunoblotting with anti-CPY antibody. The ER (p1) and mature (m) forms of CPY are indicated. Dividing line between the blots indicates blots from different gels. 
expectations, Dox-treated tetO-SAR1 cells expressing either Sar1-NES or Sar1-NESmt (Fig. 3E, labeled "SAR1-NES" or "SAR1-NESmt") were inefficient in converting the 1 form of CPY to the mature form (m), indicating that the NES moiety may partially interfere with the transport function of Sar1. As for Sar1-NESmt, since expression of Sar1-NESmt alone rescued the sar $1 \Delta$ cells (Fig. 2B), the ability of Sar1NESmt to mediate transport seems sufficient to maintain cell viability. Furthermore, Dox treatment of tetO-SARI cells expressing Sar1-NESmt displayed normal morphology of the nuclear envelope (Fig. 3C, D), suggesting that the integrity of the nuclear envelope is not associated with vesicle transport. With respect to Sar1-NES, even Doxuntreated tetO-SAR1 cells expressing Sar1-NES accumulated the $\mathrm{p} 1$ form of CPY for reasons that are yet unknown. This dominant negative effect of Sar1-NES on CPY trafficking implies that Sar1-NES is at least partially activated by Sec12 in vivo. It is possible that the interaction between the NES portion of the Sar1-NES and the nuclear export machinery sterically interferes with the transport activity. Nonetheless, Dox-untreated tetO-SAR1 cells expressing Sar1-NES showed normal nuclear envelope morphology (Fig. 3C, D), indicating again that the vesicle trafficking ability and proper nuclear envelope formation are not always interdependent. Together, these results indicate that Sar1 in the nucleus participates in the formation of the nuclear envelope, presumably independent of the COPII vesicle generation at the ER.

\section{Discussion}

We demonstrate here that Sar1 plays a vital role within the nucleus in maintaining cell viability. Our biochemical fractionation and microscopy data demonstrate that Sar1, localized in the nucleus, is predominantly associated with the inner nuclear membrane (Fig. 1). When nuclear localization of Sar1 was impeded by the attachment of a bulky fluorescent protein tag, mCherry, or fusion with NES, a severe growth defect was observed (Fig. 2). It may be assumed that the Sar1-mCherry protein was partially cleaved to release the mCherry portion of the fusion protein, thus generating a small amount of nuclear import-competent Sar1. This may explain why Sar1-mCherry is less effective than Sar1-NES in decreasing cell viability. By using conditional genetic depletion, we further showed that Sar1 expression in the nucleus is required for the proper formation of the nuclear envelope (Fig. 3). Our data demonstrated that the expression of functional Sar1 in the cytosolic fraction alone is not enough to maintain normal nuclear envelope morphology. We conclude that Sar1 plays an essential role inside the nucleus, which may relate to the proper formation of the nuclear envelope.

While our previous study provided functional evidence that the Sec23 homolog Nel1 is associated with Sar1 func- tion, the chromosomal deletion of the NEL1 gene did not cause any apparent growth defect (Kodera et al., 2014). If the localization of Sar1 in the nucleus is essential for the vegetative growth of yeast cells, why does the deletion of its GAP, i.e. Nel1, in the nucleus not affect cell viability? One simple explanation for this is that the presence of either additional Nell homologs or entirely different enzymes may provide some redundancy in function. Alternatively, the Nel1 GAP activity toward Sar1 within the nucleus may not be a critical requirement for cell survival and the exact functional relationship between Sar1 and Nel1 requires further investigation.

How does Sar1 reaches the inner nuclear membrane? The perinuclear ER is structurally continuous with the outer nuclear membrane. Therefore, one model to explain the targeting of Sar1 from the peripheral ER to the inner nuclear membrane proposes that Sar1, with a molecular mass of $21.5 \mathrm{kDa}$, slips through via the nuclear pore complexes that span the inner and outer nuclear membranes. In this model, localization of Sec12-GEF to the inner nuclear membrane is not necessary to recruit Sar1. Alternatively, soluble Sar1GDP may be passively transported through the nuclear pore complex to enter the nucleoplasm. In such a model, ERlocalized Sec12 can be laterally relocated between the perinuclear ER and the inner nuclear membrane via the nuclear pore complex, since the cytosolic domain $(38 \mathrm{kDa})$ is also small enough to pass through the nuclear pore complex. While both models are feasible, further experiments need to be conducted to determine how Sar1 translocates to the nucleus.

In conclusion, our results provide novel information regarding the function of Sar1 in the nucleus. Although Sar1 has been shown to act as a key component in the regulation of COPII-mediated protein transport from the ER, we provide evidence here indicating that Sar1 is required both in the cytosol and inside the nucleus for yeast cell growth. The fact that the perinuclear ER staining pattern of Sar1 cannot be distinguished from the inner nuclear membrane staining may explain why a nuclear-localized Sar1 has remained undiscovered for a quarter of century since Sar1 was first reported (Nakano and Muramatsu, 1989). Further investigation may provide insights into the physiological relevance of Sarl function in the nucleus.

Acknowledgments. We are grateful to the members of the Sato laboratory for helpful discussions. This work was supported in part by the Grant-inAid for Scientific Research (16K07340 and 17KT0105) from the Japanese Ministry of Education, Culture, Sports, Science and Technology. This work was also supported in part by the Naito Foundation and the Takeda Science Foundation.

\section{References}

Barlowe, C. and Schekman, R. 1993. SEC12 encodes a guaninenucleotide-exchange factor essential for transport vesicle budding from the ER. Nature, 365: 347-349.

Barlowe, C.K. and Miller, E.A. 2013. Secretory protein biogenesis and 
traffic in the early secretory pathway. Genetics, 193: 383-410.

Bi, X., Corpina, R.A., and Goldberg, J. 2002. Structure of the Sec23/24Sar1 pre-budding complex of the COPII vesicle coat. Nature, 419: 271277.

Bi, X., Mancias, J.D., and Goldberg, J. 2007. Insights into COPII coat nucleation from the structure of Sec23.Sar1 complexed with the active fragment of Sec31. Dev. Cell, 13: 635-645.

Gomez-Navarro, N. and Miller, E. 2016. Protein sorting at the ER-Golgi interface. J. Cell Biol., 215: 769-778.

Huang, M., Weissman, J.T., Beraud-Dufour, S., Luan, P., Wang, C., Chen, W., Aridor, M., Wilson, I.A., and Balch, W.E. 2001. Crystal structure of Sar1-GDP at 1.7 A resolution and the role of the NH2 terminus in ER export. J. Cell Biol., 155: 937-948.

Kakoi, S., Yorimitsu, T., and Sato, K. 2013. COPII machinery cooperates with ER-localized Hsp40 to sequester misfolded membrane proteins into ER-associated compartments. Mol. Biol. Cell, 24: 633-642.

Kalderon, D., Roberts, B.L., Richardson, W.D., and Smith, A.E. 1984. A short amino acid sequence able to specify nuclear location. Cell, 39: 499-509.

Kodera, C., Yorimitsu, T., Nakano, A., and Sato, K. 2011. Sed4p stimulates Sarlp GTP hydrolysis and promotes limited coat disassembly. Traffic, 12: 591-599.

Kodera, C., Yorimitsu, T., and Sato, K. 2014. Sec23 Homolog Nel1 Is a Novel GTPase-activating Protein for Sar1 but Does Not Function as a Subunit of the Coat Protein Complex II (COPII) Coat. J. Biol. Chem., 289: 21423-21432.

Kosugi, S., Hasebe, M., Matsumura, N., Takashima, H., Miyamoto-Sato, E., Tomita, M., and Yanagawa, H. 2009. Six classes of nuclear localization signals specific to different binding grooves of importin alpha. $J$. Biol. Chem., 284: 478-485.

Lee, M.C., Orci, L., Hamamoto, S., Futai, E., Ravazzola, M., and Schekman, R. 2005. Sarlp N-terminal helix initiates membrane curvature and completes the fission of a COPII vesicle. Cell, 122: 605-617.

Miller, E., Antonny, B., Hamamoto, S., and Schekman, R. 2002. Cargo selection into COPII vesicles is driven by the Sec24p subunit. EMBO J., 21: 6105-6113.

Miller, E.A., Beilharz, T.H., Malkus, P.N., Lee, M.C., Hamamoto, S., Orci, L., and Schekman, R. 2003. Multiple cargo binding sites on the COPII subunit Sec24p ensure capture of diverse membrane proteins into transport vesicles. Cell, 114: 497-509.

Mossessova, E., Bickford, L.C., and Goldberg, J. 2003. SNARE selectivity of the COPII coat. Cell, 114: 483-495.

Nakano, A., Brada, D., and Schekman, R. 1988. A membrane glycoprotein, Sec12p, required for protein transport from the endoplasmic reticulum to the Golgi apparatus in yeast. J. Cell Biol., 107: 851-863.

Nakano, A. and Muramatsu, M. 1989. A novel GTP-binding protein, Sarlp, is involved in transport from the endoplasmic reticulum to the Golgi apparatus. J. Cell Biol., 109: 2677-2691.

Okuno, Y., Imamoto, N., and Yoneda, Y. 1993. 70-kDa heat-shock cognate protein colocalizes with karyophilic proteins into the nucleus during their transport in vitro. Exp. Cell Res., 206: 134-142.

Saito, Y., Yamanushi, T., Oka, T., and Nakano, A. 1999. Identification of SEC12, SED4, truncated SEC16, and EKS1/HRD3 as multicopy suppressors of ts mutants of Sar1 GTPase. J. Biochem. (Tokyo), 125: 130137.

Sato, K. and Nakano, A. 2005. Dissection of COPII subunit-cargo assembly and disassembly kinetics during Sar1p-GTP hydrolysis. Nat. Struct. Mol. Biol., 12: 167-174.

Tabata, K.V., Sato, K., Ide, T., Nishizaka, T., Nakano, A., and Noji, H. 2009. Visualization of cargo concentration by COPII minimal machinery in a planar lipid membrane. EMBO J., 28: 3279-3289.

Vida, T.A., Graham, T.R., and Emr, S.D. 1990. In vitro reconstitution of intercompartmental protein transport to the yeast vacuole. J. Cell Biol., 111: $2871-2884$

Wen, W., Meinkoth, J.L., Tsien, R.Y., and Taylor, S.S. 1995. Identification of a signal for rapid export of proteins from the nucleus. Cell, 82: 463473.

Yorimitsu, T. and Sato, K. 2012. Insights into structural and regulatory roles of Sec16 in COPII vesicle formation at ER exit sites. Mol. Biol. Cell, 23: 2930-2942.

Yoshihisa, T., Barlowe, C., and Schekman, R. 1993. Requirement for a GTPase-activating protein in vesicle budding from the endoplasmic reticulum. Science, 259: 1466-1468.

(Received for publication, July 3, 2019, accepted, August 5, 2019 and published online, August 9, 2019) 